\title{
Extracellular Synthesis of Silver Nanoparticles by Soil Isolate of Staphylococcus sp.
}

\author{
Toshika Mishra ${ }^{1 *}$, Shrutidhara Kakoti ${ }^{1 *}$, Bannhi Das ${ }^{1 \#}$ \\ 1. Department of Biotechnology, Mount Carmel College, Autonomous, Bengaluru, 560001, India \\ *Both the authors have contributed equally \\ Corresponding author: bannhi.das@gmail.com
}

\begin{abstract}
Nanoparticles are congregate of atoms ranging in size from $1-100 \mathrm{~nm}$. There exists three basic ways of nanoparticle synthesis- physical synthesis, chemical synthesis and green synthesis. The limitations for the first two methods led to the development of green synthesis which is economical and less harmful for the environment. Green synthesis uses living forms like bacteria, virus, fungi, algae as well as plant extracts. Nanoparticles produced from bacteria are based on the principle of microbial metal reduction. The synthesis process can either be extracellular or intracellular depending on the type of microorganisms used. The present study highlights the extracellular synthesis of silver nanoparticles (AgNPs) from soil isolates of Pseudomonas sp. and Staphylococcus sp. The bacterial cultures after isolation were acclimatized in increasing concentration of silver nitrate in growth media and subsequently used for synthesis process. The nanoparticles formed by both the organisms were characterized by UV-Visible Spectrophotometer, FTIR Analysis and Particle size analysis. The absorption maxima for Pseudomonas sp. and Staphylococcus $s p$. cell free extracts were obtained at 410 and $400 \mathrm{~nm}$ which approximately corresponds to the size range of 20-40 nm and 10-20 nm, respectively. Though there are several reports related to Pseudomonas $s p$. being used for nanoparticle synthesis, there are very few reports related to the Staphylococcus sp. being used for the same. The study clearly indicates the capability of Staphylococcus $s p$. to form nanoparticles with physical properties comparable to the Pseudomonas $s p$.
\end{abstract}

\section{Introduction}

Nanoparticles are congregate of atoms in the size range of $1-100 \mathrm{~nm}$. Research on nanoparticle is currently an area of intense scientific research, due to its potential applications in biomedicine, biosensors, environmental bioremediation, optical and electronic fields, and use in drug and gene delivery.

The nanoparticles have unique physical and chemical properties like electronic, optical and chemical. These may be different from each component in bulk. Thus, at nanoscale the behaviour of materials is different from that at larger scales. The principal parameters of nanoparticles are shape, size, surface characteristics and inner structure. One of the important characteristics of nanoparticles is Surface Plasmon Resonance (SPR). It is a collective oscillation of conduction band electrons in metal nanoparticles excited by the electromagnetic nature of incident light. The principle behind biosensors based on nanoparticles is SPR characteristic (Schrand et al. 2008, Michael et al. 2014).

Nanoparticles are categorized into 2 types based on composition- Organic (which includes carbon nanoparticles) and inorganic (which includes metallic nanoparticles) (Kalpana et al. 2018). Examples of metals used as nanoparticles include magnesium, copper, gold, zinc, silver, selenium, etc. out of all these metallic nanoparticles, silver nanoparticles are widely used because of their antimicrobial properties and effectiveness. Thus, it is widely used as anti-bacterial agent (Ragaa et al. 2019, Noura et al. 2018).

There are three basic approaches to synthesise nanoparticles- physical synthesis, chemical synthesis and green synthesis. Although the conventional physical and chemical methods of synthesis are quite efficient, but they have major drawbacks like high cost, release of hazardous or toxic by-products in the environment, etc. This led to the development of an eco-friendly, economical and easy approach called 'green synthesis' Green synthesis uses living forms like bacteria, virus, fungi, algae as well as plant extracts. The nanoparticles synthesised by this approach are highly stable and well characterised.

Nanoparticles produced from bacteria are based on the principle of microbial metal reduction. Some bacterial species are capable of reducing metal ions to metals at nanometer scale. Optimization of processes of bacterial synthesis can result in obtaining nanoparticles with desired morphologies and controlled sizes by a fast and clean method. Bacteria possess remarkable ability to reduce heavy metals ions and so are efficient choices for nanoparticle synthesis. Some species tend to develop the ability to resort to specific defence mechanisms to overcome stresses such as heavy metal toxicity (Siavash Iravani. 2014). Nanoparticle formation can be either extracellular or intracellular depending on the type of microorganisms used. The reduction of metal ion to metal nanoparticle is carried out by NADH dependent reductase enzyme of the bacteria (Xiangqian Li et al. 2011). 
Advantages of using bacteria for nanoparticle synthesis are- use of clean, non-toxic, biocompatible and eco-friendly method; cost effective, safe and sustainable; and also bacteria are easy to handle and manipulate. On the other hand, the disadvantages are-culturing of micro-organisms is time consuming; difficult to have control over size, shape and crystallinity; and also the particles are not mono-dispersed and rate of production is low (Mandal et al. 2006)

\section{Materials and Methods}

\section{Isolation and characterisation of bacterial strain}

The bacterial strain was isolated from the soil sample collected from Mount Carmel College, Bangalore. The soil sample was serially diluted in $0.85 \%$ saline. To obtain isolated colonies 40 microlitres of the serially diluted sample was then spread on nutrient agar (NA) containing peptone $5.0 \mathrm{~g}$, HM peptone B\# $1.5 \mathrm{~g}$, yeast extract $1.5 \mathrm{~g}$, sodium chloride $5.0 \mathrm{~g}$ and agar $15.0 \mathrm{~g}$ in $1000 \mathrm{~mL}$ of distilled water. Furthermore, pure cultures were obtained from the isolated colonies using streaking method.

Biochemical characteristics of the strains were tested to identify the genus of bacteria.

\section{Acclimatization of strain}

The pure culture of Pseudomonas sp. and Staphylococcus $s p$. were inoculated in nutrient broth (containing peptic digest of animal tissue $5.0 \mathrm{~g}$, sodium chloride $5.0 \mathrm{~g}$, beef extract $1.5 \mathrm{~g}$ and yeast extract $1.5 \mathrm{~g}$ in $1000 \mathrm{~mL}$ of distilled water) with increasing concentrations of $\mathrm{AgNO}_{3}(0.005 \mathrm{mM}, 0.01 \mathrm{mM}, 0.05 \mathrm{mM}, 0.1 \mathrm{mM}, 0.5 \mathrm{mM}$, $1 \mathrm{mM})$.

\section{Synthesis of silver nanoparticles}

Theacclimatized Pseudomonasand Staphylococcus strain were cultured in $100 \mathrm{ml}$ of nutrient broth in $250 \mathrm{~mL}$ Erlenmeyer flasks. The flasks were incubated overnight at $37^{\circ} \mathrm{C}$ in a shaker incubator (150 rpm). The contents of the flasks were then centrifuged to obtain supernatant. The supernatant of both the samples was taken in a clean, sterilized flasks and $\mathrm{AgNO}_{3}$ solution was added at $1 \mathrm{mM}$ final concentration. This was further incubated at $150 \mathrm{rpm}$ and $37^{\circ} \mathrm{C}$ for 72 hours. The synthesis of nanoparticles was monitored by detecting visual change in the colour of the medium.

\section{Characterization of silver nanoparticles}

\subsection{UV-visible spectrophotometer}

The bio-reduction of silver ions to metallic silver was detected and recorded using UVvisible spectrophotometer (Systronics UV-visible spectrophotometer 117) from 360 to $480 \mathrm{~nm}$.

\subsection{Fourier transform-infrared (FT-IR) spectroscopy}

The FT-IR spectral studies using Perkin Elmer Spectrum Two Fourier transform infrared spectrometer (St. Joseph's College, Trichy) establish the functional groups that cap the formed silver nanoparticles. The spectra were recorded within the wavelength of $400 \mathrm{~cm}^{-1}$ and $4000 \mathrm{~cm}^{1}$. The shifts in the spectra at different region was analysed.

\subsection{Particle Size Distribution (DLS)}

The particle size of the silver nanoparticles was measured using Micromeritics Nano Plus (St. Joseph's College, Trichy) to determine the distribution of size of the particles in suspension. This was done to analyse the size of the particles and their dispersity in the suspension.

\section{RESULTS}

\section{Isolation and characterisation of bacterial strain}

After three times purification of colonies on NA agar followed by biochemical analysis indicated that the strains isolated belong to Pseudomonas $s p$. and Staphylococcus sp. (Table 1)

Table 1. biochemical test results for Staphylococcus sp. and Pseudomonas sp.

\begin{tabular}{|l|c|c|c|}
\hline SI.no. & $\begin{array}{c}\text { Biochemical } \\
\text { tests }\end{array}$ & $\begin{array}{c}\text { Result of } \\
\text { Strain 1 } \\
\text { (Staphylococ- } \\
\text { cus sp.) }\end{array}$ & $\begin{array}{c}\text { Result of } \\
\text { Strain 2 } \\
\text { (Pseu- } \\
\text { domonas sp.) }\end{array}$ \\
\hline 1. & Gram staining & $\begin{array}{c}\text { Gram positive } \\
\text { cocci }\end{array}$ & $\begin{array}{c}\text { Gram nega- } \\
\text { tive rods }\end{array}$ \\
\hline 2. & Amylase & Negative & Negative \\
\hline 3. & Catalase & Negative & Positive \\
\hline 4. & Oxidase & Negative & Negative \\
\hline 5. & Citrate & Positive & Positive \\
\hline 6. & Sulphur reduction & Negative & Negative \\
\hline 7. & Indole & Negative & Negative \\
\hline 8. & Motility & Positive & Negative \\
\hline 9. & Methyl red & Positive & Negative \\
\hline 10. & Voges-Proskauer & Negative & Negative \\
\hline 11. & Triple Sugar & $\begin{array}{l}\text { Glucose fer- } \\
\text { mentation, } \\
\text { gas produc- }\end{array}$ & $\begin{array}{c}\text { Glucose fer- } \\
\text { mentation, } \\
\text { no gas pro- } \\
\text { tion }\end{array}$ \\
\hline
\end{tabular}

\section{Acclimatization of organisms}

Soil isolates of Pseudomonas sp. and Staphylococcus sp. were inoculated in $10 \mathrm{~mL}$ of nutrient broth containing $0.005 \mathrm{mM}, 0.01 \mathrm{mM}, 0.05 \mathrm{mM}, 0.1 \mathrm{mM}$, $0.5 \mathrm{mM}$ and $1 \mathrm{mM} \mathrm{AgNO}_{3}$ and grown for $24 \mathrm{hrs}$. After 24 hrs, growth was observed only in the tubes containing $0.005 \mathrm{mM}, 0.01 \mathrm{mM}$ and $0.05 \mathrm{mM} \mathrm{AgNO}_{3}$. The $0.05 \mathrm{mM}$ culture was further used to inoculate broths containing higher concentration of silver nitrate $0.1 \mathrm{mM}, 0.5 \mathrm{mM}$ and $1 \mathrm{mM}$ respectively. Finally, bacterial cultures capable of 
growing in $1 \mathrm{mM} \mathrm{AgNO}$ containing media was obtained (Fig 1 and 2). The strains capable of growing at $1 \mathrm{mM}$ $\mathrm{AgNO}_{3}$ concentration was further used for the synthesis of silver nanoparticles. Acclimatization of organisms from soil isolates was possible after sequential incubation to higher concentrations of silver nitrate from the lower concentration samples. Most of the previous studies were conducted on bacteria isolated from sources rich in heavy metals; however, this study was conducted using soil isolates.(Fig 1 and Fig 2).

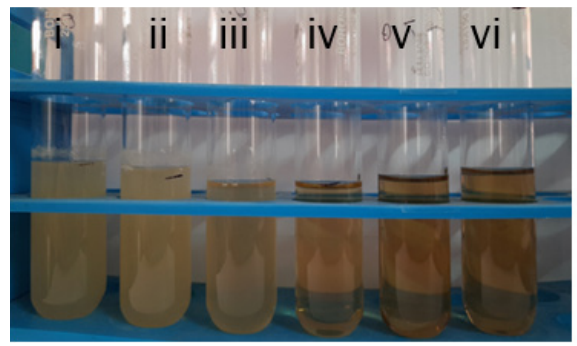

1(a)

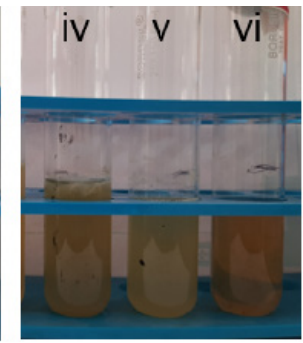

1(b)
Figure 1 Acclimatization of Pseudomonas sp. with increasing strength of silver nitrate. (a) Culture of Pseudomonas $\mathrm{sp}$ in increasing strength of $\mathrm{AgNO}$ (i) $0.005 \mathrm{mM}$, (ii) $0.01 \mathrm{mM}$, (iii) $0.05 \mathrm{mM}$, (iv) $0.1 \mathrm{mM}$ and (v) $0.5 \mathrm{mM}$ (vi) $1 \mathrm{mM}$ (b) Culture of Pseudomonas sp in increasing strength of $\mathrm{AgNO} 3$ (iv) $0.1 \mathrm{mM}$ and (v) $0.5 \mathrm{mM}$ (vi) $1 \mathrm{mM}$

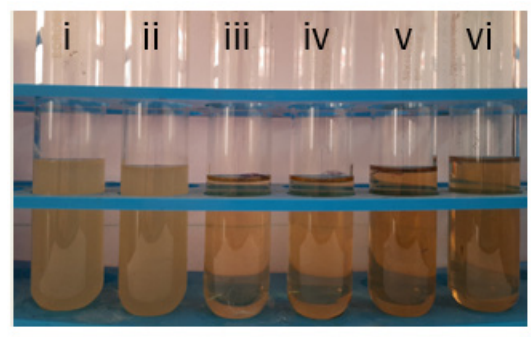

2(a)

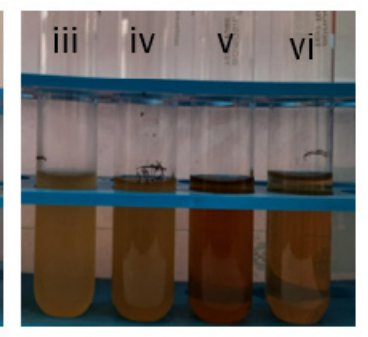

2(b)
Figure 2. Acclimatization of Staphylococcus sp. with increasing strength of silver nitrate (a) Culture of Staphylococcus sp in increasing strength of $\mathrm{AgNO} 3$ (i) $0.005 \mathrm{mM}$, (ii) $0.01 \mathrm{mM}$, (iii) $0.05 \mathrm{mM}$, (iv) $0.1 \mathrm{mM}$ and (v) $0.5 \mathrm{mM}$ (vi) $1 \mathrm{Mm}$ (b) Culture of Staphylococcus $\mathrm{sp}$ in increasing strength of $\mathrm{AgNO} 3$ (iv) $0.1 \mathrm{mM}$ and (v) $0.5 \mathrm{mM}$ (vi) $1 \mathrm{mM}$

\section{Extracellular synthesis of Ag Nanoparticles from the acclimatized cultures}

Synthesis of silver nanoparticles (AgNPs) were carried out using $24 \mathrm{hrs}$ cell free extract. The formation of nanoparticles was initially confirmed by the change in colour of the medium from light yellow to brown after $72 \mathrm{hrs}$. The cell free extracts were incubated with $\mathrm{AgNO}_{3}$ at a final concentration of $1 \mathrm{mM}$. Development of brown colour was observed in the cell free extract of Pseudomonas sp. and Staphylococcus sp. (Fig 3 and 4) after 72 hours incubation at 150 rpm; for both the organisms. Heat killed cell free extract was used as control for the experiment. This colour transition implies the biotransformation of silver ions to silver atom indicating synthesis of silver nanoparticles. The change to brown colour is due to the excitation of surface plasmon vibrations (SPV) in the particles. Though the isolates used in this study were from the normal soil, the process of acclimatization in Silver nitrate containing medium have enabled them to withstand Silver nitrate and which in turn most probably contributes towards the synthesis of the nanoparticles too.(Fig 3 and Fig 4)

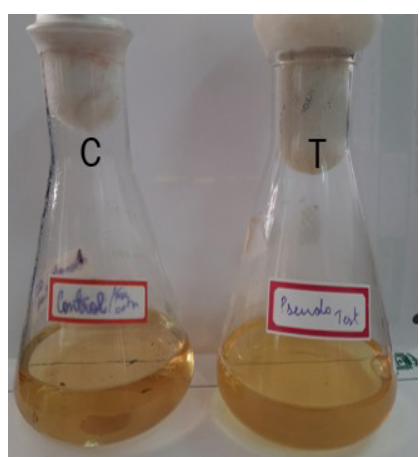

$3(a)$

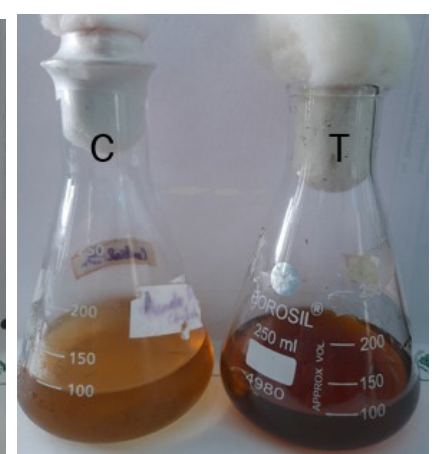

3(b)
Figure 3. Synthesis of silver nanoparticles from Pseudomonas sp. (a) day 0 - cell free extract (b) day 3 - cell free extract after incubation for 72 hours

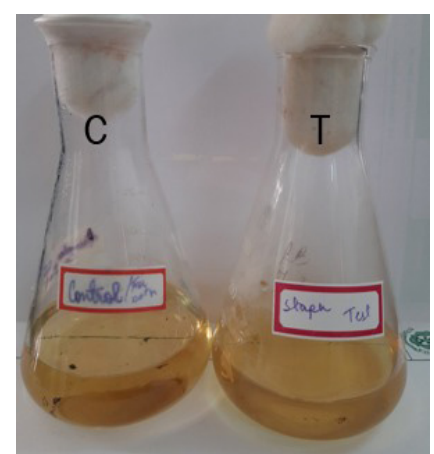

4(a)

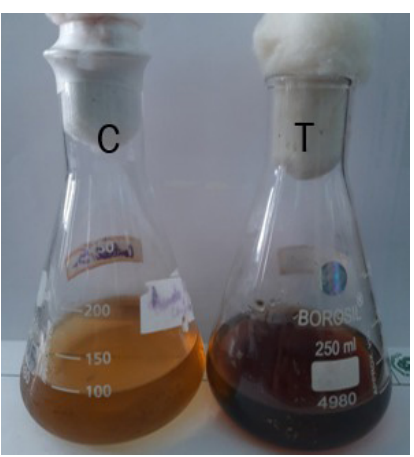

4(b)
Figure 4. Synthesis of silver nanoparticles from Staphylococcus sp. (a) day 0-cell free extract (b) day 3- cell free extract after incubation for 72 hours

\section{Characterization of silver nanoparticles}

\subsection{UV-visible spectrophotometer}

The appearance of brown colour indicates the formation of silver nanoparticles (Hina et al. 2018). The nanoparticles formed were confirmed by UV-Visible spectroscopy. During the process of nanoparticle formation reduction of silver ions takes place. The silver nanoparticles mostly exhibit absorption between 400450nm because of Surface Plasmon Resonance (SPR) (K. Anandalakshmi et al. 2016). The nanoparticles exhibit conspicuous spectral characteristics according to the Surface Plasmon Resonance (SPR) due to mutual vibrations of the free electrons resonance with light wave which is further influenced by the size and shape of the synthesized silver nanoparticles (Ragaa et al. 2019). (Table 2) (Fig 5) 


\subsection{Fourier transform-infrared (FT-IR) spectroscopy}

Table 2. Absorbance values of cell free extracts of Pseudomonas sp. and Staphylococcus sp. after 72 hours incubation

\begin{tabular}{|c|c|c|}
\hline $\begin{array}{c}\text { Wavelength } \\
\text { (in nm) }\end{array}$ & $\begin{array}{c}\text { Absorbance } \\
\text { (Pseu- } \\
\text { domonas sp.) }\end{array}$ & $\begin{array}{c}\text { Absorbance } \\
\text { (Staphylo- } \\
\text { coccus sp.) }\end{array}$ \\
\hline 360 & 1.22 & 2.03 \\
\hline 370 & 1.38 & 2.34 \\
\hline 380 & 1.50 & 2.51 \\
\hline 390 & 1.63 & 2.72 \\
\hline 400 & 1.75 & 2.88 \\
\hline 410 & 1.84 & 2.94 \\
\hline 420 & 1.79 & 2.98 \\
\hline 430 & 1.68 & 2.96 \\
\hline 440 & 1.53 & 2.89 \\
\hline 450 & 1.37 & 2.80 \\
\hline 460 & 1.18 & 2.67 \\
\hline 470 & 1.01 & 2.53 \\
\hline 480 & 0.85 & 2.32 \\
\hline
\end{tabular}

For AgNPs synthesised from Pseudomonas sp. and Staphylococcus sp., peak was observed at $410 \mathrm{~nm}$ and 420nm, respectively (Fig 5 (a) and (b)). The optical properties of silver nanoparticles synthesised depend on its diameter. Smaller particles have a peak near $400 \mathrm{~nm}$. Whereas, larger particles have broader peaks towards larger wavelengths (Paramelle et al. 2014). The UV- Visible spectra for Staphylococcus $\mathrm{sp}$. cell free extract corresponds to a size range of 10-20 nm (Paramelle et al. 2014).

FTIR is used for providing information on the local microenvironment of the organic molecules on the surface of nanoparticles. They are carried out to identify various functional groups (Pannerselvam et al. 2015). FTIR measurements were carried out for the samples (Pseudomonas sp. and Staphylococcus sp) to identify the possible biomolecules, that are probably responsible for capping and efficient stabilization of the metal nanoparticles synthesized by the cell free extracts. The results of the FTIR analysis shows different stretches of bonds at different peaks corresponding to different functional groups. For the Pseudomonas $s p$. the peaks were obtained at $3446.08 \mathrm{~cm}^{-1}, 1636.70$ $\mathrm{cm}^{-1}, 1217.13 \mathrm{~cm}^{-1}, 1120.69 \mathrm{~cm}^{-1}$ and $660.57 \mathrm{~cm}^{-1}$ (Fig 6(a)) corresponding to $\mathrm{O}-\mathrm{H}$ stretching (intra-molecular bonded), $\mathrm{C}=\mathrm{C}$ stretching, $\mathrm{C}-\mathrm{O}$ stretching, $\mathrm{C}-\mathrm{N}$ stretching and $\mathrm{C}-\mathrm{Br}$ stretching, respectively. For the Staphylococcus $s p$. the peaks were obtained at $3444.75 \mathrm{~cm}^{-1}, 1634.38$ $\mathrm{cm}^{-1}$ and $666.66 \mathrm{~cm}^{-1}$ (Fig 6(b)) corresponding to O-H
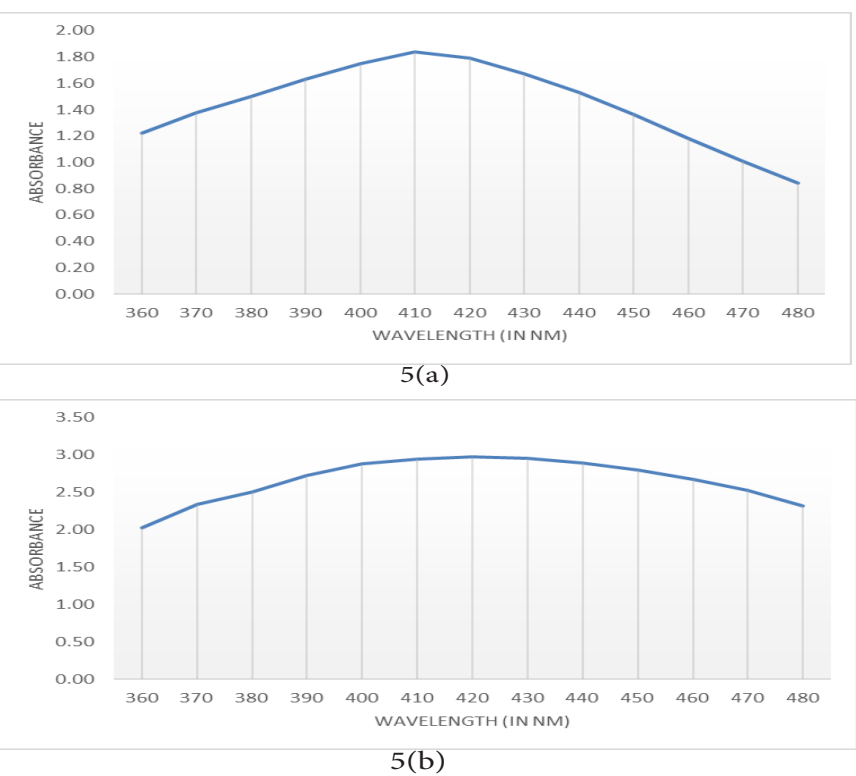

Figure 5. UV-Visible spectrophotometric analysis (a) Absorbance of Pseudomonas sp. at 72 hours (b) Absorbance of Staphylococcus sp. at 72 hours.

stretching (intra-molecular bonded), $\mathrm{C}=\mathrm{C}$ stretch and $\mathrm{C}=\mathrm{C}$ bending, respectively. A broad peak at 3446.08 $\mathrm{cm}^{-1}$ and $3444.75 \mathrm{~cm}^{-1}$ is characteristic of O-H stretch of hydrogen bonded alcohol. The peaks observed at $1636.70 \mathrm{~cm}^{-1}$ and $1634.38 \mathrm{~cm}^{-1}$ are assigned $\mathrm{C}=\mathrm{C}$ stretching alkene (Preetha et al. 2013). The frequency of the peaks of this bond are slightly lower for the extract of silver nanoparticles from Staphylococcus $s p$. than that of Pseudomonas sp. Bending alkene gave a peak at $666.66 \mathrm{~cm}^{-1}$. Further the peaks at $1217.13 \mathrm{~cm}$ 1, $1120.69 \mathrm{~cm}^{-1}$ and $660.57 \mathrm{~cm}^{-1}$ correspond to C-O bond of vinyl ether, $\mathrm{C}-\mathrm{N}$ bond of aromatic amines and $\mathrm{C}-\mathrm{Br}$ bond of halo compounds, respectively. Thus, FTIR analysis revealed the presence of alcohol and alkene vinyl ether in the cell free extract of synthesised silver nanoparticles. Furthermore, binding of nanoparticles to the amino acids in the proteins and enzymes of the bacteria, leads to change in protein conformation and is detected at $1500-1800 \mathrm{~cm}^{-1}$. Binding of nanoparticles to the phosphate groups of nucleic acids in bacteria modifies the structure and conformation of DNA or RNA, detectable in the range $600-1200 \mathrm{~cm}^{-1}$ (Fatemeh et al. 2016). The FTIR analysis, shows different biochemical properties on the basis of chemical bonds, in bacteria. (Fig 6)

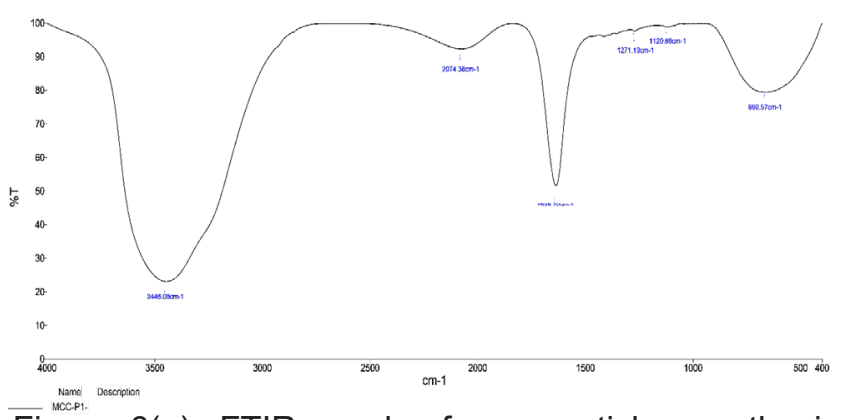

Figure 6(a). FTIR graph of nanoparticles synthesised from Pseudomonas sp. 


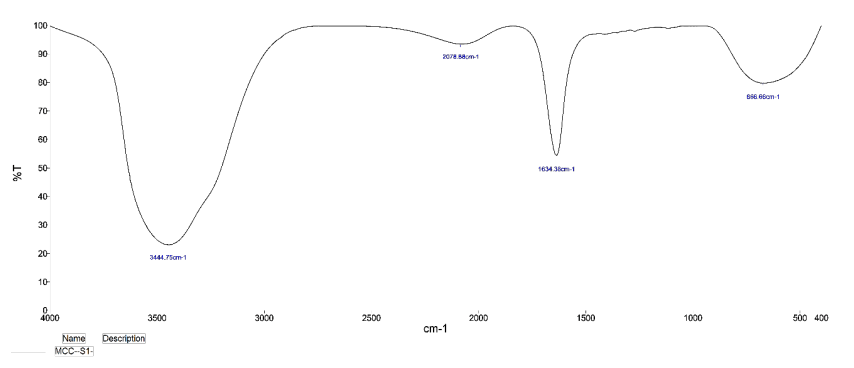

Figure 6(b). FTIR graph of nanoparticles synthesised from Staphylococcus sp.

\subsection{Particle Size Distribution (DLS)}

DLS is a method depending on the scattering of light upon interaction with particles of different size (Xi-Feng Zhang et al. 2016). This property is utilised in determining the physicochemical property of nanoparticles synthesised by different methods. DLS is a non-destructive method and provides information about the average size of the nanoparticles dispersed in a liquid medium. Different species of bacteria synthesise different ranges of nanoparticles, generally ranging from $1 \mathrm{~nm}-200 \mathrm{~nm}$ (Ayesha A. 2017). The synthesis of the nanoparticles in this study was carried in a liquid medium, which was directly subjected to DLS in order to determine the size of the nanoparticles. The average size of AgNPs synthesised from Pseudomonas sp. and Staphylococcus $s p$. was $188.4 \mathrm{~nm}$ and $364.5 \mathrm{~nm}$, respectively. However, the AgNPs range from 10nm-1000nm for Pseudomonas sp. and 8nm-1500nm for Staphylococcus sp. (Fig 7(a) and (b)). This wide range of size is most probably because of tendency of nanoparticles to form agglomerates over time. Furthermore, the diameters for the nanoparticles were observed to be $87.3 \mathrm{~nm}$ and $255 \mathrm{~nm}$ for Pseudomonas sp. and Staphylococcus sp., respectively. The Polydispersity index of silver NPs synthesised from Pseudomonas sp. is 0.388 and that of Staphylococcus sp. is 0.462 .

The size measured in DLS technique is a hydrodynamic diameter of a theoretical sphere that diffuses with the same speed as the measured nanoparticle. It is not only associated with the metallic core of the nanoparticles but also depends on the substances adsorbed on the surface of nanoparticles and the thickness of electrical double layer (solvation shell) moving with the nanoparticles (Emilia et al. 2013). This further depends on the substances present in colloids or on the surface of nanoparticles. ( Fig 7)

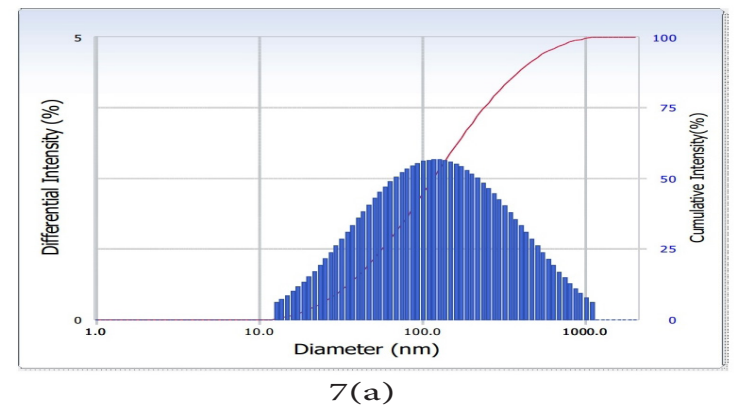

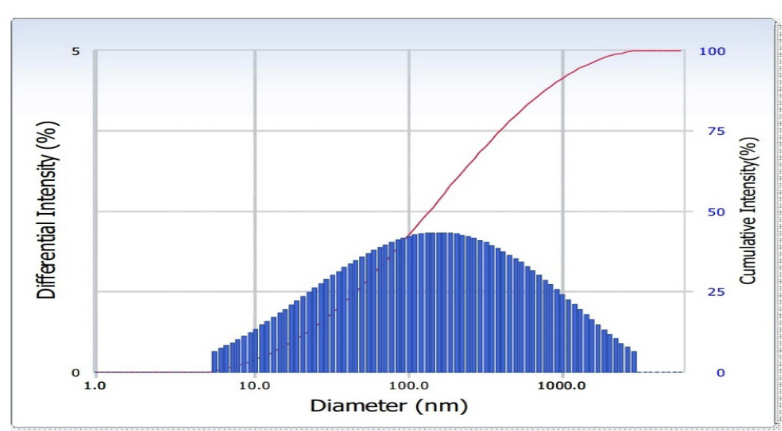

$7(\mathrm{~b})$

Figure 7. Particle size distribution (DLS) analysis (a) DLS graph of NPs synthesised from Pseudomonas sp.

(b) DLS graph of NPs synthesised from Staphylococcus sp.

\section{Discussion}

\section{Acclimatization of organisms}

Acclimatization of organisms from soil isolates was possible after sequential incubation to higher concentrations of silver nitrate from the lower concentration samples. Most of the previous studies were conducted on bacteria isolated from sources rich in heavy metals, however we conducted the study on soil isolates. Thus, by acclimatization we obtained the isolates that could adapt to the environment containing silver nitrate. The isolates could not directly grow at higher silver nitrate concentration, therefore we performed sequential inoculation at higher concentrations from acclimatized cultures with lower silver nitrate concentrations.

\section{Synthesis of Ag Nanoparticles}

Development of brown colour was observed in the cell free extract of Pseudomonas sp. and Staphylococcus sp. after 72 hours incubation at $150 \mathrm{rpm}$. This colour transition implies the biotransformation of silver ions to silver atom indicating synthesis of silver nanoparticles. The change to brown colour is due to the phenomena of surface plasmon resonance (SPR) of the particles.

\section{Characterization of nanoparticles}

\section{UV-visible spectrophotometric analysis}

The nanoparticles exhibit conspicuous spectral characteristics according to the Surface Plasmon Resonance (SPR) due to mutual vibrations of the free electrons resonance with light wave which is further influenced by the size and shape of the synthesized silver nanoparticles.

The absorption maxima for both cell free extract was obtained at $410 \mathrm{~nm}$ which approximately corresponds to the size range of $20-40 \mathrm{~nm}$. (DLS)

\section{Particle size analyzer/ Dynamic Light Scattering}




\section{- $\quad$ Fourier Transform Infrared Spectroscopy (FTIR)}

FTIR is used for providing information on the local microenvironment of the organic molecules on the surface of nanoparticles. The results of the FTIR analysis shows different stretches of bonds at different peaks. For the Pseudomonas sp. the peaks were obtained at 3446.08 $\mathrm{cm}^{-1}, 2074.38 \mathrm{~cm}^{-1}, 1636.70 \mathrm{~cm}^{-1}, 1217.13 \mathrm{~cm}^{-1}, 1120.69$ $\mathrm{cm}^{-1}$ and $660.57 \mathrm{~cm}^{-1}$ corresponding to $\mathrm{O}-\mathrm{H}$ stretching (intra-molecular bonded), $\mathrm{C}=\mathrm{C}$ stretching, respectively. For the Staphylococcus sp. the peaks were obtained at $3444.75 \mathrm{~cm}^{-1}, 2078.88 \mathrm{~cm}^{-1}, 1634.38 \mathrm{~cm}^{-1}$ and 666.66 $\mathrm{cm}^{-1}$ corresponding to $\mathrm{O}-\mathrm{H}$ stretching (intra-molecular bonded), $\mathrm{C}=\mathrm{C}$ stretching, respectively.

\section{Conclusion}

In conclusion, there has been a growing shift in interest in green synthesis of silver nanoparticles from various biological sources like plant extracts, fungi, bacteria and viruses. The soil isolates that do not belong to heavy metal rich regions can also be used to synthesize silver nanoparticles by allowing them to acclimatize to silver nitrate rich conditions. The synthesis of silver nanoparticles is confirmed by change in colour which are characterised by UV-visible spectrophotometric, FTIR and DLS analysis. In UV-visible spectrophotometric analysis we obtained a peak at $410 \mathrm{~nm}$ corresponding to nanoparticles of size range $20-40 \mathrm{~nm}$. FTIR gave information on various bonds of the nanoparticles, the stretching and bending pattern of bonds and the functional groups corresponding to the type of bond. The DLS analysis shows size and size distribution of the synthesised nanoparticles. The average size of the NPs synthesised from Pseudomonas $s p$. is $188.4 \mathrm{~nm}$ and that synthesised from Staphylococcus $s p$. is $364.5 \mathrm{~nm}$, possibly due to the formation of agglomerates by silver nanoparticles. The formation of agglomerates may reduce the activity of the particles. However, this concern can be solved with many available techniques or by perfecting the synthesis parameters so as to obtain a more effective and active nanoparticle.

\section{References}

1. Schrand AM, Braydich-Stolle LK, Schlager JJ, Dai L, Hussain SM, 2008, Can silver nanoparticles be useful as potential biological labels, Nanotechnology (volume 19, number 23)

2. Michael Holzinger, Alan Le Goff and Serge Cosnier, 2014, "Nanomaterials for biosensing applications: a review", Frontiers in Chemistry

3. V.N. Kalpana and V.Devi Rajeshwari, 2018, A review on green synthesis, biomedical applications and toxicity studies of $\mathrm{ZnO}$ nanoparticles, Bioinorganic Chemistry and Applications

4. Ragaa A.Hamouda, Mervat H. Hussein, Rasha A.
Abo-elmagd \& Salwa S. Bawazir, 2019, Synthesis and biological characterisation of silver nanoparticles derived from the cyanobacterium Oscillatoria limnetica, Nature

5. Noura El-Ahmady El-Naggar, Mervat H. Hussein \& Asmaa Atallah El-Sawah, 2018, Phycobiliproteinmediated synthesis of biogenic silver nanoparticles, characterization, in vitro and in vivo assessment of anticancer activities, Nature

6. Siavash Iravani, 2014, Bacteria in Nanoparticle Synthesis: Current Status and Future Prospects, International Scholarly Research Notices

7. Xiangqian Li, Huizhong $\mathrm{Xu}$, Zhe-Sheng Chen and Guofang Chen, "Biosynthesis of Nanoparticles by Microorganisms and Their Applications", 2011, 2 4, Vol. 2011, Journal of Nanosciences.

8. Deendayal Mandal,Mark E. Bolander, Debabrata Mukhopadhyay,Gobinda Sarkar, Priyabrata Mukherjee, "The use of microorganisms for the formation of metal nanoparticles and their application", 2006, Vol. 69, Applied Microbiology and Biotechnology !

9. Hina Singh, Juan Du, PriyankaSingh, Tae HooYi, 2018, Extracellular synthesis of silver nanoparticles by Pseudomonas sp. THG-LS1.4 and their antimicrobial application", Volume 8, Journal of Pharmaceutical Analysis

10. K.Anandalakshmi, J.Venugobal and V.Ramasamy, 2016, Characterization of silver nanoparticles by green synthesis method using Pedalium murex leaf extract and their antibacterial activity, Applied Nanoscience

11. D. Paramelle, A.Sadovoy, s.Gorelik, P.Free, J.Hobley and D.G.Fernig, 2014, A rapid method to estimate the concentration of citrate capped silver nanoparticles from UV-Visible light spectra, Analyst (issue19)

12. Pannerselvam Balashanmugam and Pudupalayam Thangavelu Kalaichelvan, 2015, Biosynthesis characterization of silver nanoparticles using Cassia roxburghii DC. aqueous extract, and coated on cotton cloth for effective antibacterial activity, International journal of Nanomedicine

13. Preetha Devaraj, Prachi Kumari, Chirom Aarti and Arun Renganathan, 2013, Synthesis and Characterization of silver nanoparticles using Cannonball leaves and their cytotoxic activity against MCF-7 cell line, Nanocomposites

14. Fatemeh Faghihzadeh, Nelson M.Anaya, Vinka 
Oyanedel-Craver, 2016, Fourier transform infrared spectroscopy to assess molecular-level changes in microorganisms exposed to nanoparticles, Nanotechnology for Environmental Engineering

15. Xi-Feng Zhang, Zhi-Guo Liu, Wei Shen and Sangiliyandi Gurunathan, 2016, Silver Nanoparticles: Synthesis, Characterization, Properties, Applications, and Therapeutic Approaches, International Journal of Molecular Sciences

16. Ayesha.A, 2017, Bacterial synthesis and applications of nanoparticles, Nano Science and Technology: An Indian journal (volume 11)

17. Emilia Tomaszewska, Katarzyna Soliwoda, Kinga Kadziola, Beata Tkacz-Szczesna, Grzegorz Celichowski, Michal Cichomski, Witold Szmaja, and Jaroslaw Grobelny, 2013, Detection Limits of DLS and UV-Vis Spectroscopy in Characterization of Polydisperse Nanoparticles Colloids, Journal of Nanomaterials 